\title{
Le Gesù, le baroque nouveau et le nouveau Montréal
}

\section{Marie Baboyant}

Volume 53, 1986

Le renouveau religieux à Montréal au XIX ${ }^{\mathrm{e}}$ siècle

URI : https://id.erudit.org/iderudit/1006973ar

DOI : https://doi.org/10.7202/1006973ar

Aller au sommaire du numéro

Éditeur(s)

Les Éditions Historia Ecclesiæ Catholicæ Canadensis Inc.

ISSN

0318-6172 (imprimé)

1927-7067 (numérique)

Découvrir la revue

Citer cet article

Baboyant, M. (1986). Le Gesù, le baroque nouveau et le nouveau Montréal. Sessions d'étude - Société canadienne d'histoire de l'Église catholique, 53, 109-120. https://doi.org/10.7202/1006973ar

Tous droits réservés @ Les Éditions Historia Ecclesiæ Catholicæ Canadensis Inc., 1986
Ce document est protégé par la loi sur le droit d'auteur. L'utilisation des services d'Érudit (y compris la reproduction) est assujettie à sa politique d'utilisation que vous pouvez consulter en ligne.

https://apropos.erudit.org/fr/usagers/politique-dutilisation/ 


\title{
Le Gesù, le baroque nouveau et le nouveau Montréal ${ }^{*}$
}

\author{
Marie BABOYANT \\ Bibliothèque municipale \\ Montréal
}

Le dimanche 11 novembre 1984, les Jésuites de Montréal ont célébré la relance de l'église du Gesù, après une restauration complète. L'événement mérite d'être signalé à plusieurs titres: le Gesù a été la chapelle annexée depuis 120 ans au Collège Sainte-Marie, la salle de théâtre au sous-sol est associée depuis lors à la vie artistique de la métropole, et enfin c'est la seule église qui soit entièrement de style baroque à Montréal.

La présente recherche n'entend pas refaire l'historique, déjà bien fait par le Père Paul Desjardins, s.j. ${ }^{1}$ Elle veut plutôt rechercher le sens de cette initiative des Montréalais de la moitié du XIXe siècle et de leur Église.

\section{I - Le retour des Jésuites à Montréal}

L'urbaniste Jean-Claude Marsan, qui est un des meilleurs connaisseurs contemporains de Montréal, montre bien l'importance de la période 18401860 pour la prise de conscience collective qui nous a donné le Montréal moderne. Il signale, en particulier, que l'arrivée d'une forte immigration de Québécois venus de diverses parties de la province donne de nouveau un caractère français à la ville ${ }^{2}$. C'est l'époque de Monseigneur Ignace Bourget (1799-1885). Le deuxième évêque de Montréal arrive avec l'intention résolue de donner une direction ferme aux catholiques de son diocèse. Il veut leur faire vivre leur foi, mais en même temps raviver leur culture. Il désire aussi que l'affection des Montréalais catholiques se tourne très concrètement vers Rome. On comprend pourquoi il décide que sa cathédrale sera une

* Nous remercions Mme Marie Baboyant et la Société historique de Montréal de nous avoir permis de reproduire cet article, paru initialement dans Les Cahiers de la Société historique de Montréal, 4 (juin 1985), p. 223-228.

1 Paul Desjardins, s.j., Le collège Ste-Marie de Montréal, vol. II, Montréal, Collège SteMarie, 1944, p. 121-152.

2 Jean-Claude Marsan, Montréal en évolution, Montréal, Fides, 1974, p. 192-193. 
réplique à échelle réduite de la basilique Saint-Pierre. On comprend aussi pourquoi il encourage les paroisses à diversifier l'architecture de leurs églises, au besoin, en faisant artificiellement un retour vers le gothique.

Parmi ces initiatives, une des plus originales est de solliciter du Général des Jésuites le retour de cette communauté, qui avait été aux origines de Montréal (17 mai 1642, avec le Père Barthélemy Vimont) et y était demeurée jusqu'à l'extinction de leur groupe (1791, avec le Père Bernard Well). Les premiers Jésuites de cette nouvelle fondation arrivèrent le 13 mai 1842. Dès le 20 septembre 1848, ils ouvrent un collège au coin des rues Saint-Alexandre et Dorchester. Il est intéressant de signalèr que ces rues ont été tracées sur l'ancienne propriété de la famille Sabrevois de Bleury dès le début du XIXe siècle ${ }^{3}$. Le collège se développe si bien que le 31 juillet 1851 , Monseigneur Bourget bénissait le nouveau collège Sainte-Marie.

Ce collège possédait naturellement sa chapelle. Elle pouvait accommoder 500 personnes. Selon la tradition des Jésuites, les élèves du collège, environ 150 au début, étaient placés dans une tribune, alors que le reste de la nef accueillait les personnes de l'extérieur. Un texte de l'époque signale que dès que la chapelle fut connue tous les bancs furent loués. Ce qui explique le besoin rapidement senti par les Jésuites tout autant que par leurs amis, de construire une église plus vaste à côté du collège.

Ce rêve avait ses antécédents. On sait que les Jésuites étaient à VilleMarie dès le 17 mai 1642. Ils assurèrent le service paroissial jusqu'en 1657. Après l'arrivée des Sulpiciens, ils repartirent pour se consacrer de nouveau à l'évangélisation des Amérindiens. En 1692, ils revinrent et construisirent une résidence et une chapelle, sur le site occupé maintenant par l'hôtel de ville, l'ancien palais de justice et le Champ de Mars. Dès 1694, ils commencèrent à enseigner le latin, sans jamais réussir, faute de subsides royaux, à constituer un collège. Cependant, leurs autres œuvres progressèrent, permettant une reconstruction de la résidence en 1703, et de la chapelle par Pierre-Janson dit Lapalme en 1719-21. Cette chapelle sera agrandie en 1742 , incendiée partiellement en 1754, restaurée en 1756 et finalement brûlée le 6 juin 1803. Elle est typiquement baroque, selon la tradition contemporaine des Jésuites français ${ }^{4}$. On comprend que l'idée de reprendre la tradition ait été fascinante soixante ans après l'incendie de l'ancienne chapelle!

3 E.-Zotique Massicotte, "Coins historiques de Montréal d'autrefois", dans Les Cahiers des Dix, Montréal, 1937, p. 138-141. L'adresse actuelle du Gesù est 1180 de Bleury.

4 La Compagnie de Jésus au Canada, 1842-1942, Montréal, 1942, p. 21; J.-C. Marsan, op. cit., p. 81. Malheureusement, les commentaires et illustrations de Pierre Moisy dans Les églises des Jésuites de l'ancienne assistance de France, Rome, Institutum Historicum S.I., 1958, vol. 1, p. 234 et vol. 2, pl. LXXI et LXXX, sont sans valeur, car elles confondent l'église des Jésuites avec la première église Notre-Dame. 


\section{II - Les avatars d'une construction}

Le chanoine Édouard Fabre, qui deviendra plus tard évêque de Montréal, semble avoir été le premier à annoncer publiquement le projet. Cette nouvelle coïncide avec l'arrivée au collège d'un recteur dynamique, le Père Louis Saché (1862-1865). Aussitôt, le Père Saché, avec le Père Rémi Tellier, supérieur de la mission jésuite, commencent à esquisser l'église projetée. Le premier plan provient d'un jeune Jésuite enthousiaste, le Père Arthur Jones, alors étudiant à Boston. Selon lui, l'église doit être gothique, inspirée par la cathédrale de Cologne. Dans la discussion, cependant, on doit tenir compte du fait que les Jésuites ne possèdent pas encore le terrain sur lequel l'église devrait être construite. Un monsieur Campbell, anglo-canadien et protestant, possède ce terrain et semble peu disposé à le vendre. La transaction sera faite cependant un an plus tard, grâce à l'intervention d'un Frère jésuite légendaire, le Frère Léon Rouillés. Un de ses amis, monsieur Olivier Berthelet, consacrera la somme de 20000 \$à l'achat de la propriété Campbell.

Pendant cette période, $\mathrm{M}^{\mathrm{gr}}$ Bourget a entendu parler du projet d'une église gothique. Il s'oppose vivement, au point que le projet est aussitôt abandonné, même après avoir été élaboré par un architecte montréalais réputé, Monsieur Lamontagne. Le recteur Saché ne trouve pas d'autre solution que de se tourner vers un autre architecte celui-là de réputation internationale, Patrick Charles Keely (1816-1896). On l'a appelé «le patriarche de l'architecture catholique aux États-Unis». Né en Irlande, immigrant fixé à Brooklyn en 1842, il domina l'architecture religieuse sur la côte est des États-Unis pendant 43 ans. On lui a attribué jusqu'à 600 églises; en fait, on a des preuves pour 150 , ce qui est déjà considérable. Au début de sa carrière, il dessine surtout du gothique, puis il évolua vers le baroque et le néo-classique. Ses constructions coûtaient cher, mais il semble que son souci de la qualité et du fini expliquent ce fait. Il a construit au moins une autre église au Canada, la cathédrale de St-Jean de Terre-Neuve ${ }^{6}$.

Le projet qu'on exprima à l'architecte Keely était le souhait de $\mathrm{M}^{\mathrm{gr}}$ Bourget: faire de l'église du collège Sainte-Marie une église typiquement romaine, qui serait dédiée au Sacré-Cœur. Les plans furent rapidement terminés, le 19 mars 1864 on commença à déblayer le terrain, le 22 mai on posa la première pierre et on commença la construction. Il n'est pas important ici de rappeler les péripéties de l'année de la construction! La correspondance du Père Tellier avec son supérieur à Rome signale que les dimensions furent sujet de controverses, que la façade faillit être en brique et

5 P. Desjardins, op. cit., p. 129-136.

6 Robert T. Murphy, "Keely, Patrick Charles», dans Macmillan Encyclopedia of Architects, New York, The Free Press, 1982, p. 556-557. 
que les tours octogonales ne furent jamais terminées, faute de fonds suffisants. Le 3 décembe 1865, l'église était bénite et ouverte au culte. La première messe ne fut pas célébrée par $\mathrm{M}^{\mathrm{gr}}$ Bourget, mais par le grand vicaire Alexis-Frédéric Truteau: $\mathrm{M}^{\mathrm{gr}}$ Bourget était à Rome depuis plusieurs mois. Montréal possédait dorénavant sa première église baroque.

\section{III - Le sens du Gesù}

Au cours de l'année 1853, un anglo-protestant montréalais peu marqué par l'œcuménisme, écrivait ceci dans un journal de New York:

«Ces dix dernières années, une silencieuse mais grave révolution succède à la stagnation d'autrefois, grâce à l'énergie d'un ordre conquérant, les Jésuites... une puissance nouvelle a surgi dans la province. Les Jésuites ont pris possession du sol et ils y restèrent. Ils imposent l'idée de stabilité par la forme substantielle de leurs édifices, par la plus habile combinaison de science, d'économie, d'éducation religieuse et séculière... Les Jésuites lancent les Canadiens dans une civilisation pleine de vie; l'effet sera de mettre le Canada au niveau de ce qu'il y a de plus distingué sur ce continent»" ${ }^{7}$.

Qu'est-ce qui provoque cette admiration et cette inquiétude? C'est l'ouverture du Collège Ste-Marie, qui est à l'origine du Gesù.

Le Gesù, c'est une église de 194 pieds de longueur sur 144 pieds de largeur dans les transepts et de 75 pieds de hauteur. Sa charpente est en bois, son extérieur en calcaire et son intérieur en plâtre. Sa façade est typiquement baroque, avec un fronton grec et une niche au centre de ce fronton. Deux étages de façade, le plus élevé fait de trois fenêtres, les plus bas de trois portes. Les tours sont obliques par rapport à la façade.

Mais c'est l'intérieur qui est plus caractéristique encore. L'espace est pratiquement d'une seule venue, avec seulement de modestes bas-côtés en dehors des hautes colonnes rondes. Les transepts coupent la nef au tiers, formant une croix. La voûte est en plein cintre et l'abside est également ronde. Et ce qui étonne c'est que toute la surface est couverte de fresques, en camaïeu, c'est-à-dire avec toutes les teintes d'une seule couleur, le brun. Tout est en trompe-l'œil, donnant l'illusion du haut-relief.

Ces fresques sont l'œuvre d'un artiste allemand devenu américain, Daniel Müller (Desjardins écrit Mulleir). Il soumet son projet, qui est approuvé le 29 juin 1865 et il commence la série de fresques qu'il terminera au printemps de 1866, quelques mois après la bénédiction de l'église. Il semble qu'un Jésuite, le Père Georges Schneider, fournit à Müller des cartons empruntés à des fresquistes de l'école de Düsseldorf.

7 Alexandre Dugré, s.j. «Le collège Sainte-Marie est fils du dévouement et du patriotisme", dans Lucien Duchaine, éd., Bourgade hier... métropole aujourd'hui, Montréal, Les Éditeurs de l'histoire de Montréal, 1951, p. 203-210. La citation est à la p. 205. 
Les anciens auteurs attribuent tout le travail au seul Daniel; plus récemment, on a remarqué qu'un frère de Daniel, Ernst, a collaboré à l'ensemble. Les fresques signées, au Gesù, portent l'initiale D. Mulleir8.

Les fresques sont conçues comme un ensemble à trois niveaux successifs. Le bas des murs représente des mystères fondamentaux de la foi chrétienne: crucifiement au-dessus du maître-autel (l'église est dédiée au Sacré-Cœur), le couronnement de Marie au-dessus de l'autel de gauche (depuis lors dédié à Notre-Dame de Liesse depuis 1877), et au-dessus de l'autel de Saint-Joseph, la glorification de ce saint par Dieu le Père. En faisant le tour de la nef, on rencontre Saint-Ignace écrivant les Exercices spirituels et toute une série de scènes de martyrs de saints Jésuites. Dans la partie supérieure des murs, au-dessus des stations du chemin de la croix (d'après des esquisses du Père Arthur Martin), le fresquiste Müller a représenté dans des médaillons les principaux saints de la Compagnie de Jésus déjà canonisés à l'époque: Ignace, François-Xavier, François de Borgia, François Régis, Louis de Gonzague, Stanislas Kostka, Jean Berchmans, Alphonse Rodriguez, Pierre Claver, Pierre Casinius, etc.

C'est cependant la voûte qui nous donne la clef de la symbolique de toute cette décoration. Au centre, le nom de Jésus est écrit en lettres d'or à la jonction de la nef et des transepts. Du côté de l'autel, l'agneau de Dieu de l'Apocalypse adoré par toute la création. Du côté de la nef centrale, l'Enfant Jésus présenté aux bergers par la Vierge Marie. À gauche, Jésus à douze ans au milieu des docteurs; à droite, la Sainte Famille avec Jésus et Joseph au travail. D'autres tableaux représentent la résurrection de Lazare, le Bon Pasteur, le retour de l'enfant prodigue: Jésus bénissant les enfants, Jésus apparaissant aux apôtres après sa résurrection et, enfin, derrière l'orgue, Jésus ressuscité entrant au ciel.

Comme on le voit, c'est toute la spiritualité de l'époque baroque et sans doute, beaucoup de la spiritualité des Jésuites que nous retrouvons ici. L'époque gothique, et d'ailleurs, l'époque byzantine et romane, tendaient à projeter la communauté croyante vers le ciel, faisant de cette terre surtout une vallée de larmes. La spiritualité nouvelle veut faire saisir au croyant que s'il admet l'incarnation de Dieu en Jésus-Christ, il doit comprendre que le sacré, que l'éternel et que l'absolument valable sont déjà ici et que l'espace sacré de l'église le représente.

Ceci nous amène à reculer notre observation jusqu'à l'ensemble du baroque lui-même. L'origine la plus vraisemblable du mot «baroque» est le portugais barroco, qui désigne une perle de forme irrégulière. Le mot

8 Gérard Morisset, La peinture traditionnelle au Canada français, Montréal, Cercle du Livre de France, 1960, p. 136-137; J. Russell Harper, Early Painters and Engravers, Toronto, University of Toronto Press, 1970, p. 233; P. Desjardins, op. cit, vol. 2, p. 205-206. 
baroque lui-même, en français, désigne depuis les environs de 1710 une idée bizarre. On rencontre le mot baroque dans le domaine architectural à partir des environs de 1788. Au début, on l'associe au bizarre, à l'abus de ce qui est étonnant, à l'extravagant. Peu à peu, on en viendra à le définir comme la forme d'art, architecture, peinture, musique, qui fait suite au maniérisme de la Renaissance. Le baroque s'étend de la Sicile à la Russie, de l'Espagne à l'Allemagne, demeurant en général dans le domaine catholique. Il s'étendra bientôt à toute l'Amérique latine. On l'a appelé "art jésuite». Cependant, les critiques contemporains refusent de voir là autre chose qu'uñe coînincideñce. Si l'art baroque accompagne les Jésuites, c'est en raison de la très grande activité missionnaire de ces derniers à la suite du Concile de Trente (15451563) et pendant tout le XVIe siècle. À l'autre extrémité de son histoire, le baroque est remplacé par le rococo et le néo-classique du XIX ${ }^{e}$ siècle 9 .

Magré toutes ces critiques, on reconnaît aujourd'hui dans le baroque plusieurs traits qui en font un fidèle reflet du monde moderne commençant: inspiration à partir des formes humaines plus que des formes végétables, comme le faisait le gothique, souci de l'intérieur beaucoup plus que de l'extérieur, usage des matériaux accessibles sur place avec, au besoin, emploi du trompe-l'œil pour donner l'illusion du matériau plus précieux ou plus rare, effort enfin de célébration de la puissance humaine, de la joie de vivre et de la profusion de la nature et de la culture. Ce sont là des traits de notre monde beaucoup plus que du Moyen Âge. On comprend déjà mieux l'enthousiasme des amis des Jésuites, y compris Monseigneur Bourget, en regardant le Gesù s'élever au nord-ouest du Vieux Montréal. On comprend également l'inquiétude des compétiteurs anglo-canadiens en constatant l'arrivée de ce symbole du monde moderne à Montréal, à la frontière de la nouvelle ville écossaise et anglaise.

À ce moment, il faut nous tourner vers l'histoire de l'architecture religieuse québécoise traditionnelle. Selon les auteurs plus anciens, lorsque nos premiers ancêtres s'installèrent au Québec au XVIIe siècle, ils apportèrent avec eux un seul style d'architecture religieuse: le style roman campagnard, tel que transmis traditionnellement par des générations d'artisans. Un expert comme Gérard Morisset a voulu en tracer les traits essentiels dans son ouvrage sur "L'architecture en Nouvelle-France» ${ }^{10}$. Nous connaissons tous, ces églises de notre province dont les traits, à première vue, sont très différents de l'art baroque: long vaisseau en maçonnerie, toiture à faîte, transepts qui servent de contreforts, abside arrondie, clocher aigü en charpente avec une ou deux lanternes. L'intérieur de ces églises est entièrement en bois et sans aucune décoration autre que de simples

9 Victor-L. Tapié, Baroque et classicisme, Paris, Plon, 1957.

10 Québec, Collection Champlain, 1949. 
moulures. On sait aujourd'hui que certains curés devenus architectes ont contribué à maintenir cette tradition en rédigeant des plans et devis si précis et si détaillés que la présence de l'architecte cessait d'être requise. Le plus connu de ces plans est l'œuvre du curé Pierre Connefroy (1752-1816), de Boucherville. Inutile d'ajouter que cet art est profondément adapté chez nous, qu'il a été pratiqué par de grands artisans comme les Bourgeau, les Lapalme, les Levasseur et les Quevillon.

Depuis une trentaine d'années, cette interprétation a été complètement remise en question, en particulier par Luc Noppen. Il devient de plus en plus évident que nos ancêtres ont apporté avec eux le baroque urbain du temps de Louis XIV. Les premières chapelles des Récollets et des Jésuites à Montréal, en sont les témoins les plus visibles. À partir des débuts, les artisans québécois ont modifié le baroque, pour l'adapter au climat et à l'entourage ${ }^{11}$. Ils l'ont rendu méconnaissable à force de l'adapter à nous et à ici. À tel point que le retour d'un baroque romain étonne et symbolise le renouveau! On comprend mieux ainsi la contradiction apparente des évaluations: pour certains, le Gesù de Montréal est la nouveauté par excellence, pour d'autres il est ce qu'il y a de plus adapté. La raison en est que notre architecture religieuse ancienne a toujours vécu du baroque, avec un ressourcement par $\mathrm{M}^{\mathrm{gr}}$ Bourget!

On peut se demander, après avoir constaté la rupture que représente l'apparition du Gesù à Montréal, au milieu du XIXe siècle, si le baroque de l'Américain Keely est véritablement du baroque. Après avoir remarqué que les constructeurs du Gesù ont utilisé les artisans locaux et les matériaux montréalais pour construire l'église, nous n'avons qu'à ouvrir un répertoire comme «Les églises des Jésuites de l'ancienne assistance de France»" ${ }^{12}$, pour constater que l'église du Gesù entre tout naturellement dans le cadre des églises baroques de la même époque en France et en Amérique latine. Par ailleurs, malgré la similitude de noms, il est bien évident que l'architecte Keely qui "connaissait le Gesù de Rome par cœur» s'en est beaucoup éloigné lorsqu'il a tracé les plans du Gesù de Montréal. Le Gesù de Rome a été créé en 1568 par l'architecte Vignola avec la contribution de Giacomo della Porta pour la façade. L'austérité que souhaitait saint Ignace pour les églises des Jésuites a été rudement contredite par le donateur, le cardinal Alexandre Farnese. Par conséquent, la richesse des matériaux (marbres, lapis-lazzuli, verrières), rien de cela ne se retrouve dans le Gesù de Montréal. Rien non plus des fresques colorées des églises jésuites romaines ne

11 Alan Gowans, "From Baroque to Neo-Baroque in the Church Architecture of Quebec", dans Culture, 10 (1949), p. 140-150; Luc Noppen, Les églises du Québec (16001850), Montréal, Éditeur officiel du Québec et Fides, 1977, p. 3-28.

12 P. Moisy, op. cit., vol. 1, p. 234. 
transperce sous les fresques monochromes de Müller. Mais le Gesù de Montréal, intuition de Monseigneur Bourget et des Jésuites revenus de l'exil, demeure un symbole puissant de l'arrivée du monde moderne et de l'église moderne à Montréal dans la moitié du XIXe siècle.

On ne peut quitter cette revue du Gesù de Montréal sans jeter un coup d'œil sur sa décoration et son mobilier. C'est en effet, à ce niveau, que l'adaptation québécoise du baroque devient la plus significative. Les artisans montréalais ont su adapter au baroque leur connaissance de la marquetterie pour réaliser les parquets splendides du chœur que la restauration vient de nous révéler. Un artisan d'ici, A.J. Pigeon, a construit les autels à retable monumental, en érable piqué. Malgré la modestie du matériau, les statues en papier-mâché des évangélistes sont une autre réalisation étonnante.

La décoration suspendue est peu importante, mais en plus du chemin de croix déjà signalé, il faut remarquer quelques grands tableaux des frères Gagliardi de Rome: saint Louis de Gonzague et saint Stanislas de Kostka recevant la communion, Jean-Enfant et Jean-Baptiste, la Fuite en Égypte. Le plus connu des frères Gagliardi, Pietro (1809-1890) travaillait à Rome. Il avait étudié sous Tommaso Minardi et s'inspirait de Canova dont il a conservé le caractère monumental et aussi la réserve classique. À l'époque où il peint les tableaux du Gesù, il décore également de fresques l'église Saint-Jérôme degli Schiavoni à Rome et peint également des fresques à Saint-Paul-hors-les-murs.

Sans vouloir faire de cette recherche une étude complète du Gesù, il est impossible de passer sous silence un élément essentiel de sa vie depuis le XIXe siècle. En 1877, un Jésuite canadien, nommé François-de-Sales Prudent Cazeau fut envoyé à Laon pour y faire sa «troisième année de noviciat». Il découvrit la dévotion à Notre-Dame de Liesse. Comme sa statue avait été brûlée en 1793, lors de la Révolution française, on conservait ses cendres dans une église de pèlerinage. Le Père Cazeau revint à Montréal en emportant une partie des précieuses cendres. Dès leur exposition à Montréal, dans une petite statue conservée au-dessus de l'autel de la Vierge Marie au Gesù, la réputation miraculeuse de cette vierge se répandit. Elle demeure toujours bien vivante et les ex-voto bien caractéristiques ajoutent un autre élément significatif des églises baroques.

Il faut remarquer que la dureté des temps empêcha les Jésuites d'alors d'ajouter de véritables verrières au Gesù. L'ingéniosité québécoise transforma des vitres ordinaires avec des papiers de couleurs. $\mathrm{La}$ restauration récente les a remplacées pour la première fois par d'authentiques verrières par les ateliers Battinger de Berthierville. 


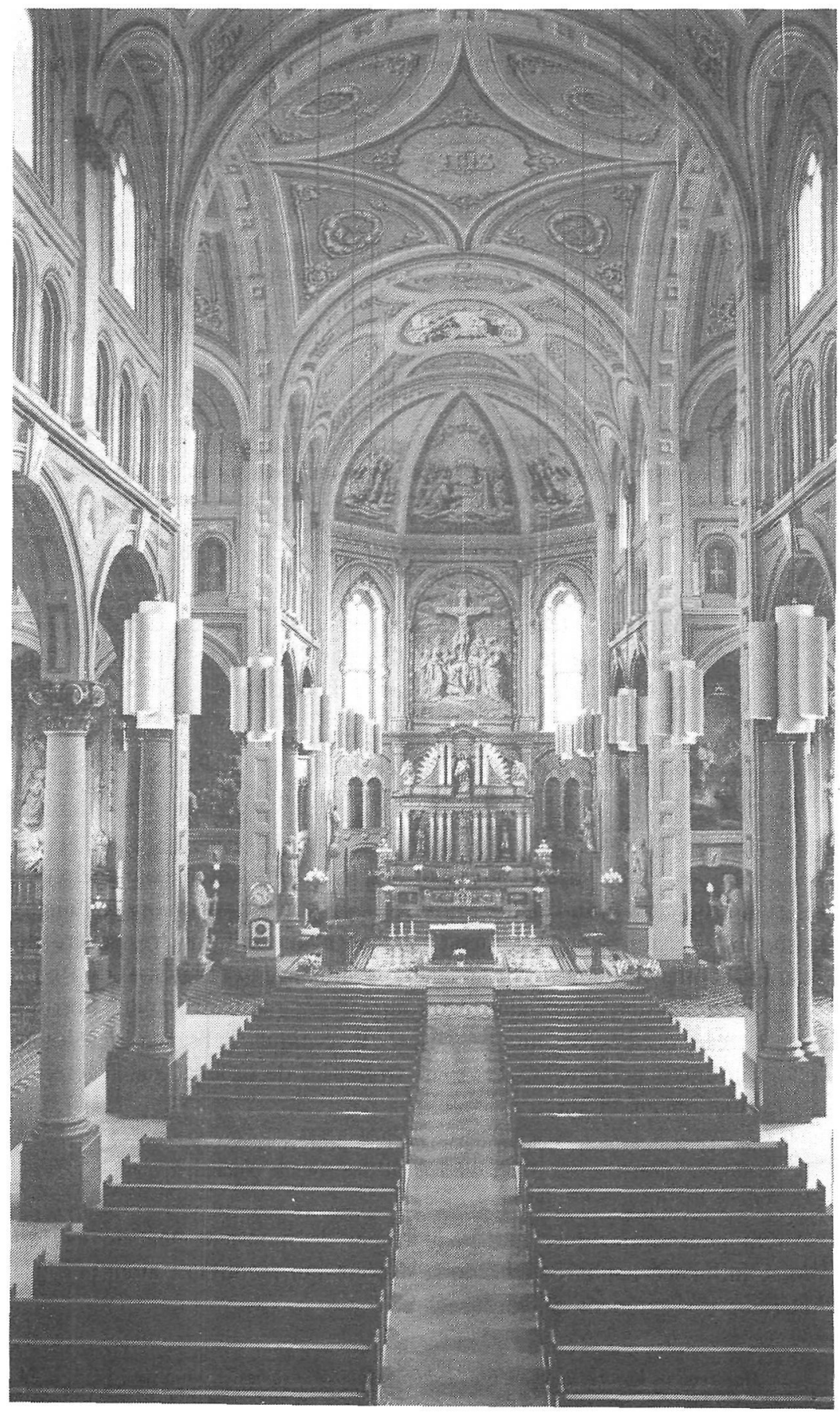




\section{IV - Ce qu'on a pensé du Gesù}

Dix ans après l'ouverture de l'église, le révérend J. Douglas Borthwick publiait une histoire de Montréal ${ }^{13}$. Il reconnaît dans le Gesù

un magnifique édifice que bien des visiteurs proclament un des plus beaux temples de l'Amérique

Après avoir décrit l'architecture et la décoration à fresques, il ajoute

outre le magnifique maître-autel, on y trouve plusieurs petits autels tous conçus et réalisés dans un style d'une richesse et d'une beauté insurpassable. Les murs sont ornés de façon splendide et enluminés d'une variété presqu'infinie de peintures et d'images bibliques. Tout cela démontre à l'évidence une grande dépense d'habileté, de patience et aussi d'argent.

En 1876, un livret anonyme bilingue intitulé Collège Ste-Marie et Église du Gesù reconnaît que

c'est sans contredit une œuvre de génie, conçue en imitation du plan de l'église du Gesù, une des plus belles basiliques de Rome... Pendant la belle saison, de nombreux visiteurs s'y succèdent à toute heure du jour, et tous en emportent un excellent souvenir et proclament le Gesù de Montréal un des plus beaux édifices religieux de l'Amérique du Nord.

On trouvera des témoignages un peu plus réservés dans l'Album souvenir du Collège Sainte-Marie de 1921, par l'architecte Marcel Pariseau ou encore dans l'Album souvenir de 1939, à l'occasion du $75^{\mathrm{e}}$ anniversaire $\mathrm{du}$ Gesù.

L'historien officiel du collège Sainte-Marie, le Père Paul Desjardins, a longuement décrit la construction et la décoration du Gesù, mais il a été réservé au point de ne pas donner son avis.

Parmi les témoignages contemporains, il est amusant de souligner celui du grand urbaniste Jean-Claude Marsan ${ }^{14}$. Son peu d'estime pour les talents artistiques de Monseigneur Bourget l'amène à une critique très sévère du Gesù tout entier. Il ne retrouve aucune ressemblance avec le Gesù de Rome «si ce n'est par une décoration en trompe-l'œil, particulièrement horrible dans le cas de l'église montréalaise». Il critique l'absence de dôme, l'obscurité quasi permanente, les tours tronquées de la façade et il termine son évaluation en disant

enfin, la seule valeur architecturale que l'on peut concéder au Gesù de la Métropole canadienne est qu'il laisse poindre une note de pittoresque qui fera la fortune des monuments victoriens à venir.

On peut soupçonner que le jugement de monsieur Marsan est, au moins, influencé par la couche de suie noire qui recouvrait alors (1974!) l'intérieur et l'extérieur du Gesù.

13 Montreal, its history, to which is added biographical sketches..., Montréal, 1875, p. 74.

14 J.-C. Marsan, op. cit., p. 220-221. 


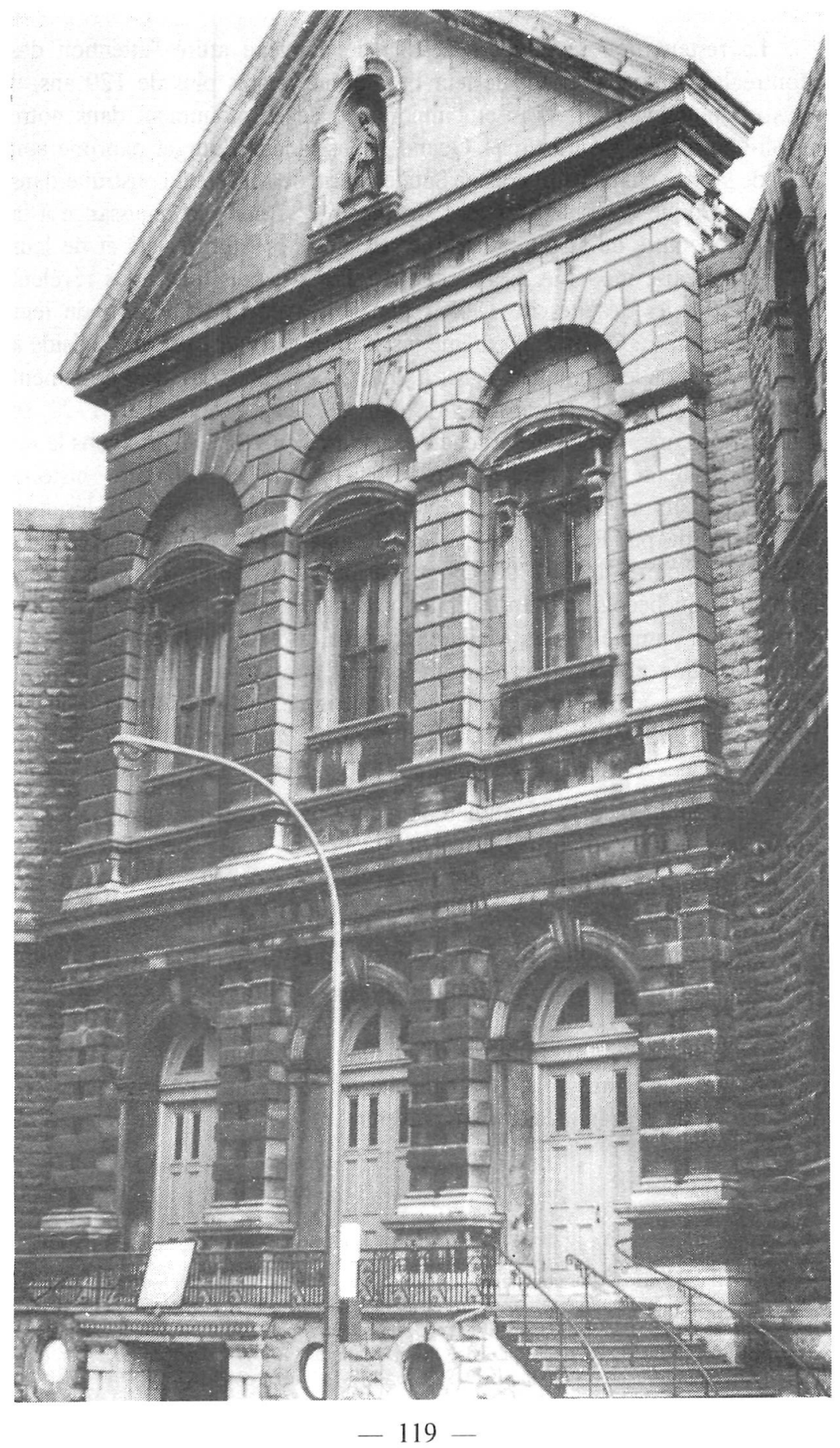




\section{V - Conclusion}

La restauration récente du Gesù a de nouveau attiré l'attention des Montréalais sur un élément de leur patrimoine. Après plus de 120 ans, il nous est plus facile de localiser l'importance de ce monument dans notre histoire spirituelle et artistique. Quand Monseigneur Bourget exprime son désir de voir la chapelle du collège Sainte-Marie de Montréal construite dans le style baroque, il voulait d'abord signifier par là une reconnaissance à la fois de la vitalité de la foi catholique romaine des Montréalais et de leur culture française moderne. Les recherches, faites depuis iors, nous révèleni qu'en réalité les Jésuites de 1865 renouaient avec une tradition de leur premier séjour au Québec. La même inspiration baroque qui avait présidé à la conception de leur première église à Québec, en 1666, qui avait également déterminé leur projet de construction dans le Vieux Montréal en 1722, se réalisait sous une forme plus proche de sa source en 1865. Nous avons là un résumé étonnant de notre histoire architecturale et de notre histoire spirituelle. Comme l'a montré Luc Noppen, le modèle d'église des Jésuites, qui ne signifie pas "art jésuite», a été dominant avec celui des Récollets pendant l'époque 1660-1700, pour ensuite se fondre, à travers une adaptation québécoise de plus en plus profonde, avec le type récollet et les plans locaux comme le plan Maillou. À partir de 1700, le baroque de style Louis XIV que nos ancêtres français avaient apporté au Québec, devient tellement nôtre qu'un bon historien de l'art comme Gérard Morisset a pu s'y méprendre. L'art religieux québécois a en réalité toujours été inspiré par le baroque. Dans le Gesù, il est rajeuni.

Si les Jésuites de 1865, au lieu de recourir à l'architecte Keely, avaient requis les services d'un constructeur québécois de l'école de Thomas Baillargé (1791-1859), ils auraient construit en baroque, mais en baroque québécois.

Cette constatation est, sans doute, après 120 ans, la meilleure explication de l'inquiétude du Montréalais anglophone que j'ai cité au début de ce texte. Il avait raison de voir dans cette construction un signe évident de la vitalité de la culture française d'ici, non seulement dans ses origines européennes, mais aussi dans sa capacité de se maintenir, d'assimiler ses richesses et de se renouveler dans l'avenir $^{15}$.

15 Il serait intéressant de poursuivre cette étude dans quelques autres églises montréalaises d'illustration baroque renouvelée, au moins en partie; en particulier: l'église Notre-Dame-deGrâce, réalisée en 1851-53 par James Ostell et Joseph Marr, l'église de Ste-Anne-de-Bellevue et la chapelle de l'Hôtel-Dieu de Montréal. Olivier Maurault, Marges d'histoire II, Montréal, Librairie d'Action Canadienne-française, 1929, p. 137-143 et p. 241-267. 